\title{
Accuracy Improvement of a Machine Kinematics for the Product Flexible Machining of Curved Extrusion Profiles
}

\author{
J. Fleischer ${ }^{1, a}$, J. P. Schmidt-Ewig ${ }^{1, b}$ \\ ${ }^{1}$ wbk Institute of Production Science, Universität Karlsruhe (TH) \\ Kaiserstraße 12, 76128 Karlsruhe, Germany \\ âFleischer@wbk.uka.de, ${ }^{\mathrm{b}}$ SchmidtEwig@wbk.uka.de
}

Keywords: Kinematics, handling, machining

\begin{abstract}
Within traffic engineering, the importance of lightweight space frame structures continuously grows. The space frame design offers many advantages for light weight construction but also brings challenges for the production technology. For example, the important requests concerning product flexibility and reconfiguration can only be achieved with a high technical effort, if current machine technology is used. For this reason, the collaborative research center SFB/TR10 investigates the scientific fundamentals of a process chain for the product flexible and automated production of space frame structures.

An important component in space frame structures are curved extrusion profiles. Within the investigated process chain, the extrusions must be machined mechanically in order to apply holes and to prepare the extrusion ends for the following welding operation. The machining is currently done by clamping the profile into a fixture and processing it within a machining center. This procedure has two disadvantages due to the complex geometry and the partially high length of the extrusion profiles: On the one hand, a complex fixture is needed for clamping the work piece [1]. On the other hand, a machining center with a large workspace and five machine axes is required [2]. Due to this, the product flexible machining with current technology is only possible with high technical and economical effort. For this reason, a new machine concept for the product flexible machining of three dimensionally curved extrusion profiles was developed at the University of Karlsruhe. In this paper, the function of the machine is explained and a prototype is presented. In addition, investigation results of the machining accuracy are shown and possibilities for improving the precision are discussed.
\end{abstract}

\section{Machine Concept for Product Flexible Machining}

Based on a systematic approach for integrating handling and machining capabilities [2], the machine concept shown in figure 1 was designed. It is build up out of three major components: An industrial robot, a parallel kinematic machine tool and a flexible clamping device which is located in between. 


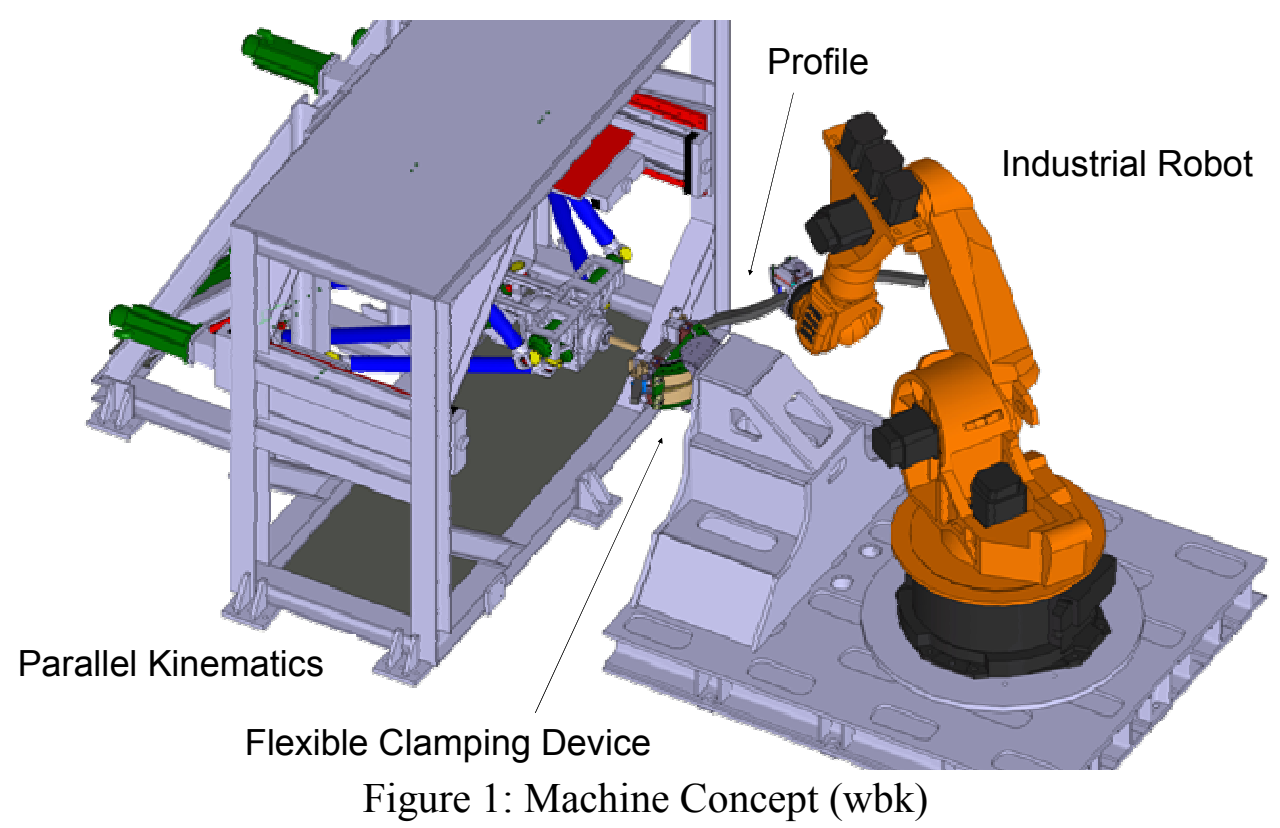

The industrial robot is used for the handling tasks which result from the process chain. In addition it also supports the machining process by moving the extrusion profile within the clamping device in order to position the workpiece area which is to be machined next to the tool center point. The machining angle can also be adjusted by turning the rear end of the profile around the clamping device. For this, the clamping unit is pivoted and possesses two rotational degrees of freedom that allow a rotation around the tool center point. After having adjusted the desired angle, the rotation axes can be blocked for reaching a high stiffness [1]. The translational movements between tool and workpiece needed for machining are executed by the parallel kinematic machine tool which was optimized for this task [3].

The machine concept several advantages. The number of required degrees of freedom of the machining kinematics can be reduced by adjusting the machining angle with the industrial robot. In addition the entire workpiece can be machined without needing a clamping fixture which was adapted to the longitudinal geometry of the extrusion. The required work space of the machining kinematics can also be reduced significantly which enables a high potential for reducing costs. Figure 2 shows the prototype of the machine concept.

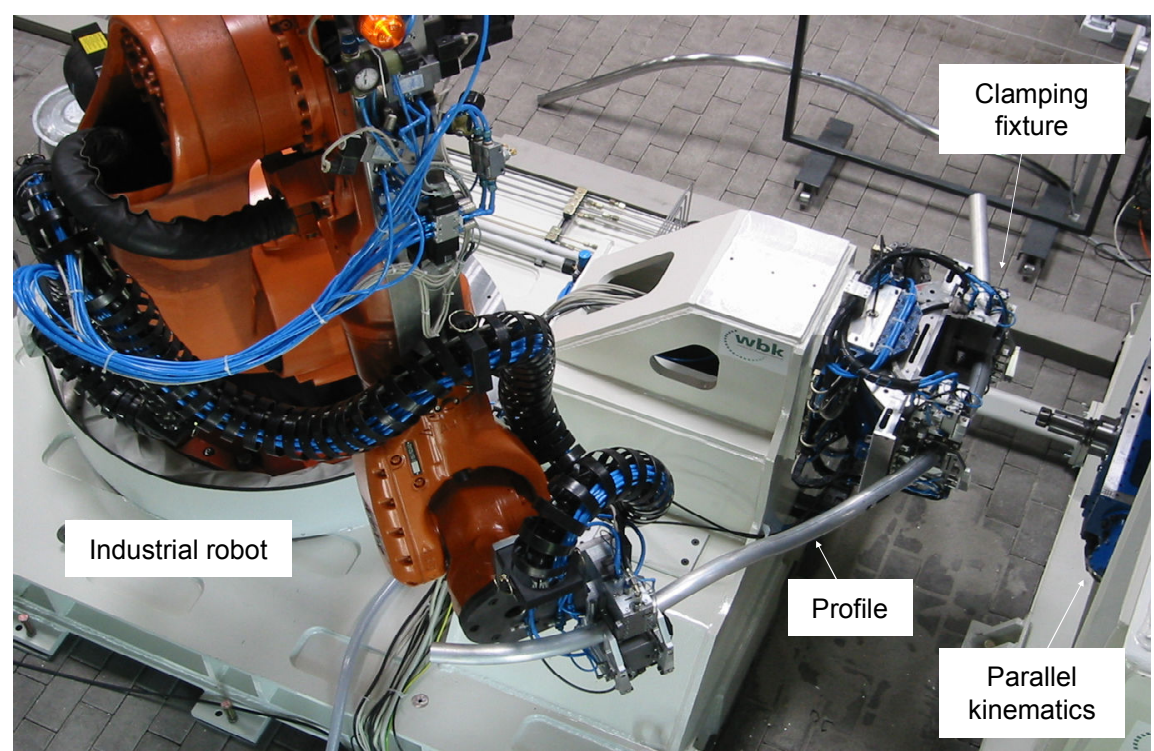

Figure 2: Prototype (wbk) 
The three main components (robot, parallel kinematics and clamping device) are controlled by the robot control (KRC2 control) and a free programmable real-time interface (dSPACE system). Figure 3 shows the architecture of the entire control system. The procedure of the machining process is defined in the robot control. With the help of this control, all movements needed for handling and positioning the workpiece are executed. After having positioned the extrusion within the clamping device, the robot control sends a digital signal to the dSPACE system. This part of the control executes the clamping action and enables the machining movements of the parallel kinematics which were defined in an NC-program previously. The dSPACE system and the frequency converters together form a cascaded position control. The superposed position control, the required coordinate transformation and the error compensation are executed in the dSPACE system while the velocity and current control is located in the frequency converters.

When the machining action is done, a digital signal is sent to the robot control in order to move the workpiece to the next machining position.

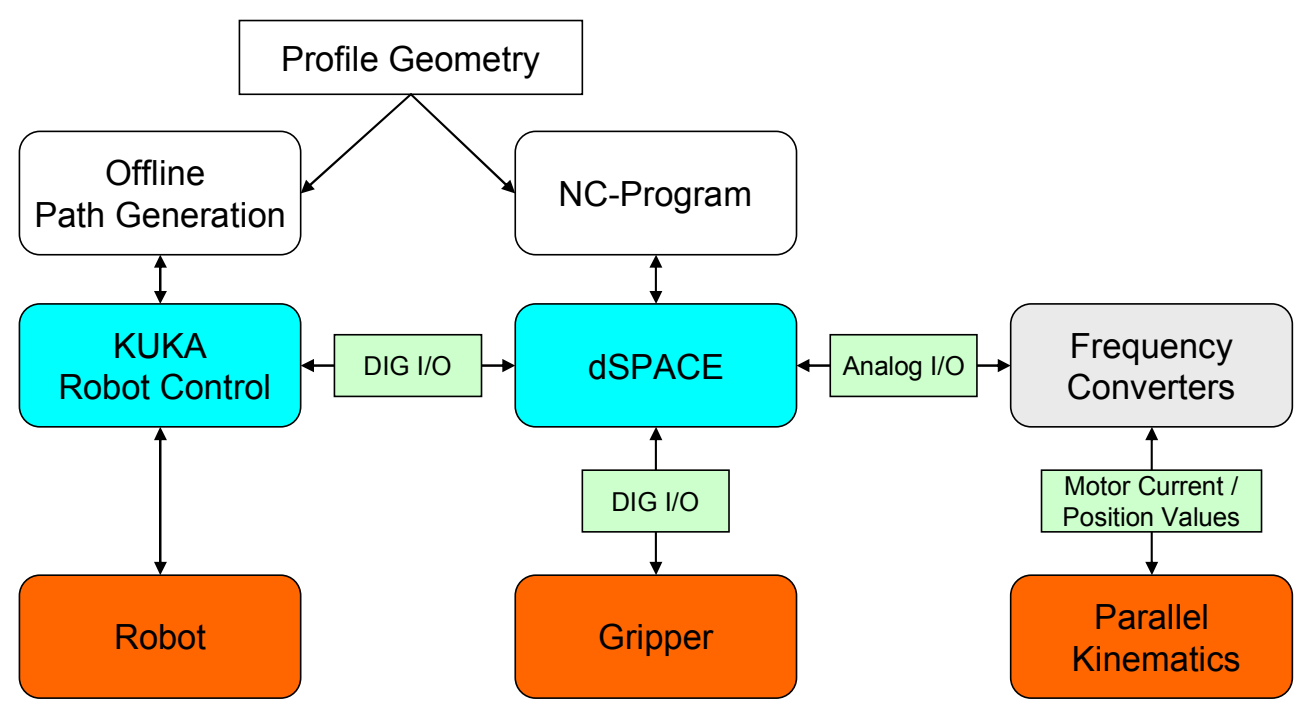

Figure 3: Control System

In order to move the extrusion profile precisely within the clamping device, a complex robot path must be generated which can not be done with conventional programming procedures. For this reason, the robot path is calculated with the help of a CAD-based kinematic model which precisely simulates the gliding movements of the extrusion on the positioning rolls of the clamping device [4]. Thus, the angular movements of the robot axes can be determined which result from a longitudinal and angular movement of the extrusion in the clamping device. The recorded data are transferred to the robot control. This method is similar to the procedure which is used within the collaborative research center for creating the tool path for the flying cutting [5].

\section{Examination of the Positioning Accuracy}

The offline path generation described above enables an easy creation of machining programs also for complex workpiece geometries. This is a prerequisite for a quick reconfiguration of the production system for new work pieces as it is necessary in a product flexible production. Due to a limited accuracy of the robot and deviations between the geometry of the kinematic model used for programming and the real machine, a limited positioning accuracy is expected. The precision of the parallel kinematics could be improved by an error compensation as described in [3], so that this part of the system does not cause major errors. Yet, the precise positioning of the workpiece within the clamping device is still a challenge. These deviations can already be limited by fixing the extrusion using positioning rolls. This way the extrusion position is always clearly defined orthogonal to the 
longitudinal workpiece axis. The remaining deviations are errors in the longitudinal position and the machining angle.

The precession which is aspired within the collaborative research area SFB/TR10 is a deviation of $\pm 0.1 \mathrm{~mm}$ on one meter workpiece length. The desired angular precision is $\pm 0.01^{\circ}$. In order to quantify the actual deviations, positioning experiments were performed. Figure 4 shows the experimental set up.

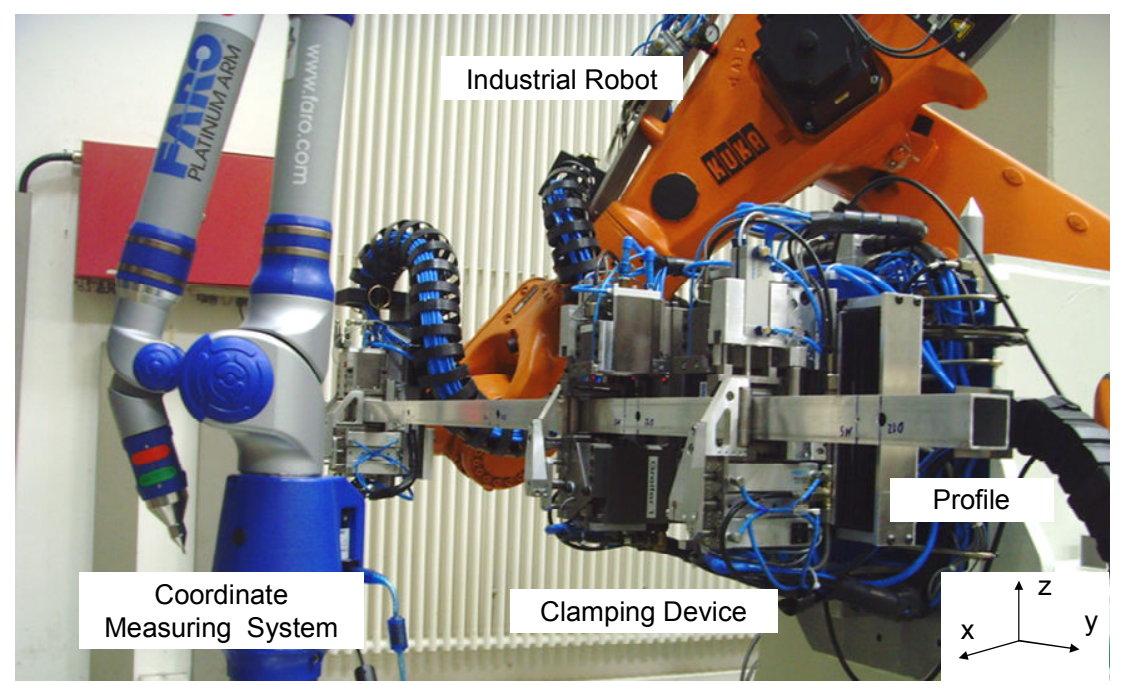

Figure 4: Experimental Set Up

The experiments initially focus on the positioning of a straight profile. Due to the fact that the error influences are the same for straight and curved extrusions, it can be assumed that the deviations for curved workpieces have the same dimensions. At first, the straight extrusion is shifted through the clamping device in five $200 \mathrm{~mm}$ steps and then moved back to the initial position. Next, the workpiece is rotated in $15^{\circ}$ steps around a total angle of $90^{\circ}$ around the turning axis of the clamping device ( $\mathrm{x}$-axis in figure 4). Finally the profile is turned $\pm 15^{\circ}$ around the tilting axis (z-axis in figure 4). In each step the deviations of the two angles (turning and tilting angle) as well as the deviation of the longitudinal workpiece position are determined with the help of a coordinate measuring system. The angular deviations can precisely be determined by measuring the orientation of the workpiece surfaces. The longitudinal error is measured with help of an index hole. The following figure shows the results of three exemplary measurements.

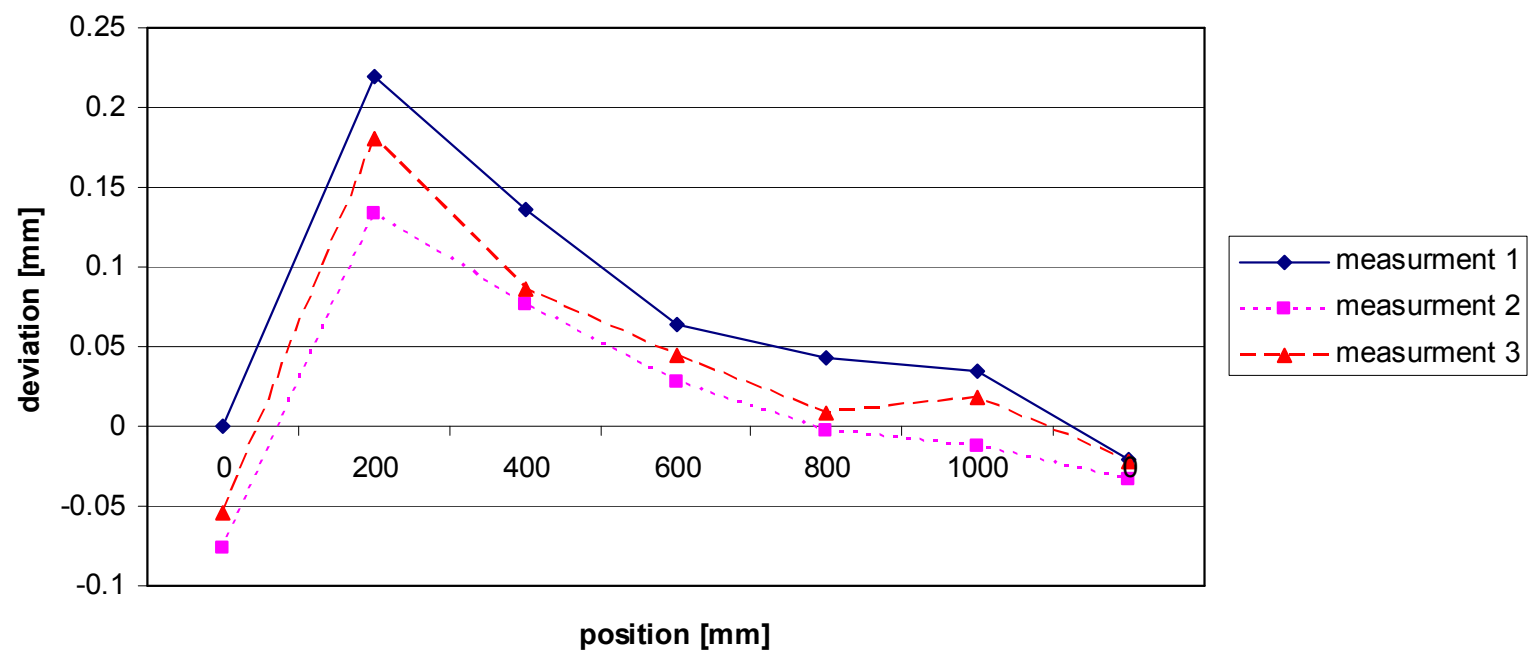

Figure 5: Longitudinal Deviations 


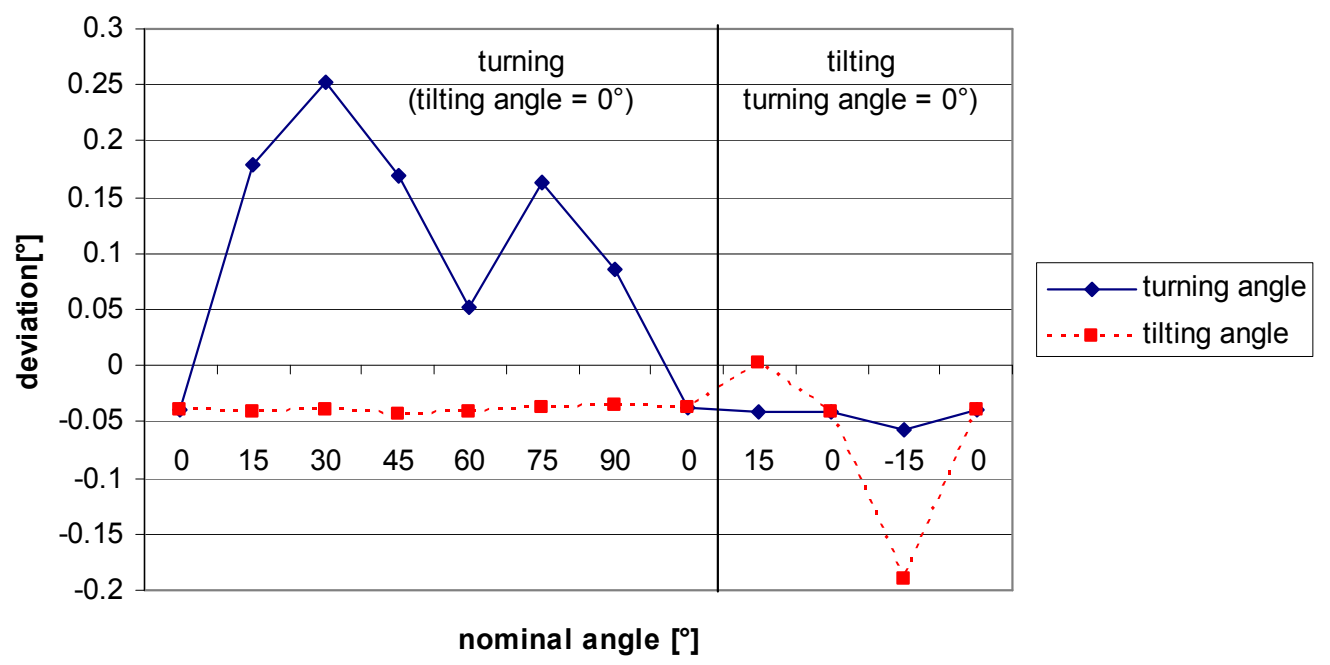

Figure 6: Angular Deviations

Figure 5 shows the longitudinal deviations of the profile. The maximum error is $0.25 \mathrm{~mm}$ and occurred after a displacement of $200 \mathrm{~mm}$. When the experiment is repeated, the error pattern shows a similar progress. Yet, an error distribution within a range smaller than $0.1 \mathrm{~mm}$ can be recognized. This distribution can be referred to the repeatability of the industrial robot which is in the range of $\pm 0.1 \mathrm{~mm}$, according to the manufacturer.

In Figure 6 the angular error is shown for different work piece orientations. The left side of the diagram refers to a rotation around the turning axis (x-axis), in the right side the profile is tilted $\pm 15^{\circ}$ around the z-axis. The maximum deviation of the turning angle is $0.25^{\circ}$ and occurs for a desired angle of $30^{\circ}$. The maximum error of the tilting angle is $0.19^{\circ}$. The repeatability of the rotation has turned out to be very good. This is also shown by the similar values for the different zero-positions (value 1, 8, 10 and 12 in figure 6). The errors follow a non-linear pattern for both rotations. In addition, it can be seen that the turning and tilting angle have only a small interdependency. According to this observation, the two angles can be corrected independently. In conclusion, the absolute deviations must still be improved in order to reach the aspired accuracy.

\section{Approach for Accuracy Improvement}

The major influences for the observed positioning errors can be summarized as follows:

- geometrical deviations between the kinematic model and the real machine

- deviating workpiece geometry

- clamping errors

- insufficient accuracy of the industrial robot

The geometrical deviations between the machine prototype and the kinematic model used for programming were eliminated as far as possible by measuring the position and orientation of the clamping device and the robot using a coordinate measuring system. The programming model was adapted to the deviations afterwards. A compensation of the influence of the workpiece geometry is more problematic. In order to position the workpiece precisely in the clamping device, the entire kinematic chain consisting of robot and extrusion profile must perform a high accuracy. In order to reach this aim, an improved accuracy of the robot in not sufficient. In addition, the geometry of every single workpiece must be determined and the robot path must be adapted adequately. Due to the fact that this procedure has a high technical and temporal effort, a different approach was chosen in order to enhance the machining accuracy.

The deviations of the workpiece position and orientation are detected by sensors directly located in the clamping device and can afterwards be compensated by a correction movement of the robot. 
In order to detect the angular deviations of the extrusion, two rotary encoders were integrated into the clamping device. For a detection of the longitudinal errors, different approaches were investigated at the Universität Karlsruhe $(\mathrm{TH})$ [6]. A procedure which is based on an image processing system seems to be very promising. This procedure is described more detailed in the paper "An approach to accuracy improvements in the flexible machining of curved profiles" of this issue.

After having detected the deviations, they can be reduced by correcting the position of the robot. For this, it is necessary to calculate the corrected robot coordinates in dependency on the sensor data and the actual position of the extrusion profile. Due to the complex geometry of the extrusion profiles this calculation seems to be very complex in the first instance. The following geometrical considerations show how the corrected coordinates are calculated for deviations of the turning angle, the tilting angle and the longitudinal position. It is shown, that the corrected position can be determined independently from the extrusion geometry.

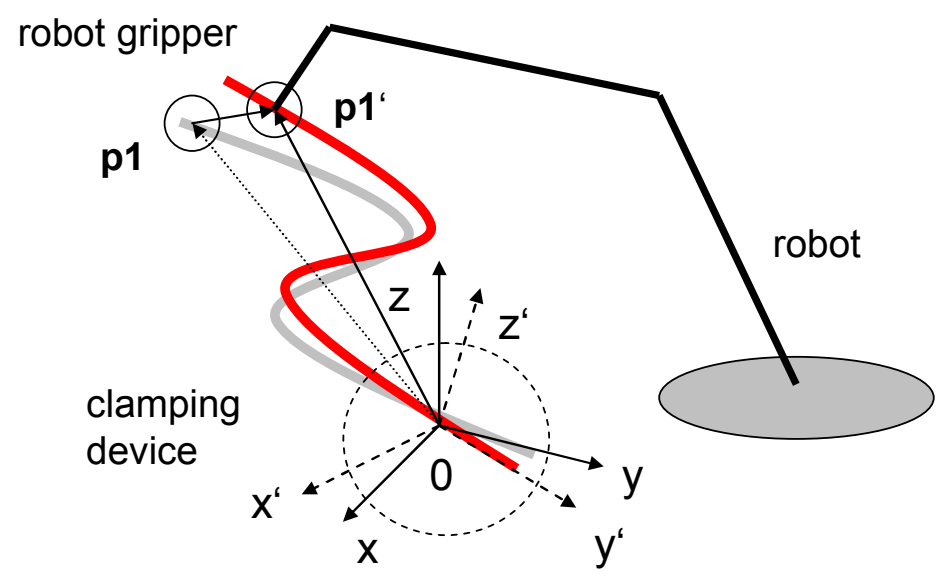

Figure 7: Rotation of the Extrusion

Figure 7 shows the arrangement of the rotational axes and the industrial robot. The point of origin (0) marks the center point of rotation of the clamping device. The vector $\mathbf{p} 1$ describes the distance between the point of origin and the center point of the robot gripper. The coordinates of $\mathbf{p} 1$ in relation to the center point of rotation can be accessed from the robot control. For this, the real center point of the clamping device and the robot gripper were measured and set as base and tool coordinate system in the robot control. Thus, the base coordinate system of the robot has the same position and orientation as shown in figure 7. The rotational axes of the clamping device allow the workpiece to turn around the $\mathrm{x}$ - and $\mathrm{z}$-axis, whereas a turning around the $\mathrm{x}$-axis changes the orientation of the z-axis. A rotation of the extrusion $(\mathbf{p} 1 \rightarrow \mathbf{p} \mathbf{1}$ ') can therefore be described with a rotation of the $\mathrm{x}$-axis and a following rotation of the $\mathrm{z}$-axis. The rotation around the $\mathrm{x}$-axis can be calculated with help of the rotary matrix $\mathbf{R x}$, whereas $\alpha$ represents the corresponding turning angle [7].

$R x=\left[\begin{array}{ccc}1 & 0 & 0 \\ 0 & \cos (\alpha) & \sin (\alpha) \\ 0 & -\sin (\alpha) & \cos (\alpha)\end{array}\right]$

The rotation around the z-axis (tilting angle $\beta$ ) can be described with $\mathbf{R z}$ : 
$R z=\left[\begin{array}{ccc}\cos (\beta) & \sin (\beta) & 0 \\ -\sin (\beta) & \cos (\beta) & 0 \\ 0 & 0 & 1\end{array}\right]$

The total rotary matrix $\mathbf{R M}$ can be calculated by multiplying $\mathbf{R x}$ and $\mathbf{R z}$ :

$R M=R z \cdot R x$

If an angular deviation of the $\mathrm{x}$-axis $(\mathrm{d} \alpha)$ is measured, it can be compensated by a rotation around the $\mathrm{x}$-axis of the base coordinate system. In practice, the robot can perform such an action with a circular movement. However, the end point of the path must be known for this. The point can be calculated independently from the workpiece geometry, as shown in figure 8 .

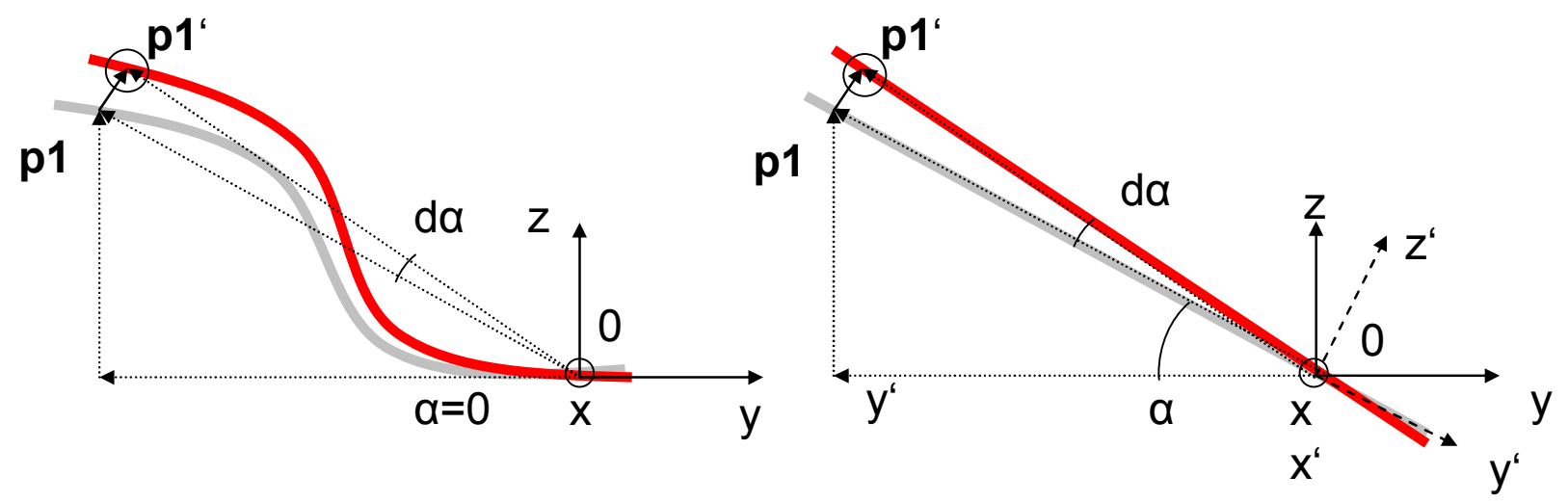

Figure 8: Angular Compensation

The end point of the path p1' can be determined with help of the actual position of the robot gripper $\mathbf{p} 1$ and the angular deviation $(\mathrm{d} \alpha)$. It can be calculated with the rotary matrix $\mathbf{R x}$ as follows:

$p 1^{\prime}=R x^{T} \cdot p 1$

The robot paths which are needed for compensating the deviation d $\alpha$ shown in figure 8 are the same for both extrusion geometries. If a deviation $d \beta$ of the tilting angle (z-axis) needs to be compensated, it must be considered that the tilting axis is changed with a rotation of the $\mathrm{x}$-axis ( $\mathrm{z} \rightarrow$ z'). Even though the robot gripper has the same position for both extrusions in the figure above, the orientations of tilting axes are different because the right extrusion was rotated about the angle $\alpha$. For compensating the deviation $\mathrm{d} \beta$, a second end point (p1") must be calculated. This can be done with help of the turning angle $\alpha$, by transforming the actual position $\mathbf{p} \mathbf{1}$ (which is only known in the base coordinate system) into the rotated coordinate system ( $\left.\mathrm{x}^{\prime}, \mathrm{y}^{\prime}, \mathrm{z}^{\prime}\right)$, performing a rotation around the $\mathrm{z}$-axis about $\mathrm{d} \beta$ and, in a last step, transforming the new point back into the original system. The equitation for calculating the coordinates of $\mathrm{p}^{\text {" }}$ ' in the base coordinate frame $(\mathrm{x}, \mathrm{y}, \mathrm{z})$ is the following:

$p 1^{\prime \prime}=R x^{T} \cdot R z^{T} \cdot R x \cdot p 1$

Longitudinal deviations must theoretically be compensated by a movement which follows the extrusion contour. For a straight extrusion, a linear path is sufficient. Yet, for a curved extrusion the robot gripper must theoretically also follow a curved path for avoiding tensions in the kinematic system. If the curve radius of the workpiece contour is large compared with the longitudinal displacement, the compensation path can be very well approximated with a linear motion. This is clarified in figure 9: 


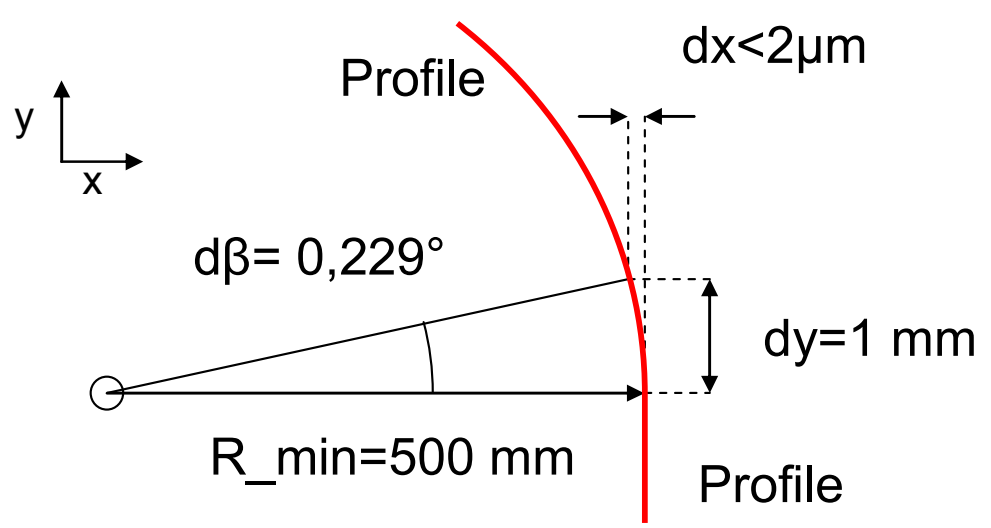

Figure 9: Approximation of the Longitudinal Compensation Path

In figure 9 the curve radius of the workpiece is assumed to be $500 \mathrm{~mm}$, which corresponds to the minimal workpiece radius of the extrusion profiles regarded in the collaborative research center. The deviation which is to be compensated is assumed to be $1 \mathrm{~mm}$. This is a realistic value corresponding to the previously performed measurements. If the compensation path is approximated with a linear motion, the deviation along the $\mathrm{x}$-axis is smaller than $2 \mu \mathrm{m}$. According to this, small longitudinal deviations can be compensated with the procedure shown in figure 10 .

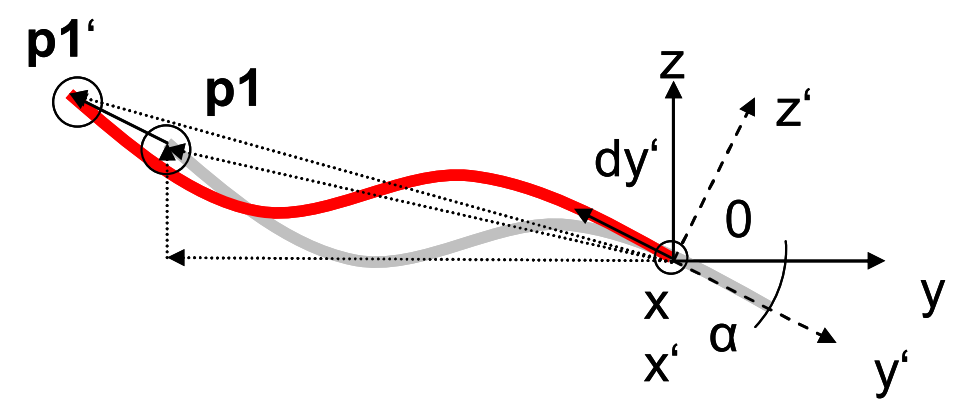

Figure 10: Longitudinal Compensation

A measured deviation can be send to the robot control as a scalar value (a). The required position p1' can be calculated with the orientation of the clamping device ( $\alpha$ and $\beta$ ), the corresponding rotary matrix $\mathbf{R M}$ and the actual position $\mathbf{p 1}$ as follows:

$p 1^{\prime}=p 1+R M^{T} \cdot d y=p 1+d y^{\prime}$

The vector $\mathbf{d y}$ can be calculated with the deviation a and the unit vector ey:

$d y=a \cdot e y$

If the workpiece has a small curve radius, the compensation of a longitudinal deviation can lead to small angular errors. According to this, it is reasonable to compensate the longitudinal deviations first and correct the orientation afterwards. In a next step, the rotational axes of the clamping device can be blocked in order to ensure a correct orientation while machining the workpiece. Based on the calculations shown above, a sub program was integrated into the robot control which can be accessed by the main program after each positioning step and performs an automatic correction of the workpiece orientation. With this procedure, new positioning measurements of the workpiece were performed. 


\section{Positioning Accuracy with Compensation}

The positioning accuracy with compensation was measured by new experiments. In order to guarantee the function of the compensation procedure for all eight space segments of the base coordinate system, the positioning experiment described above was extended. The turning angle is changed stepwise from $15^{\circ}$ to $-195^{\circ}$. In every step, a tilting angle of $-15^{\circ}, 0^{\circ}$ and $15^{\circ}$ is adjusted. After each positioning step, the sub program for error compensation is performed. Figure 11 shows the deviations before and after the compensation procedure.

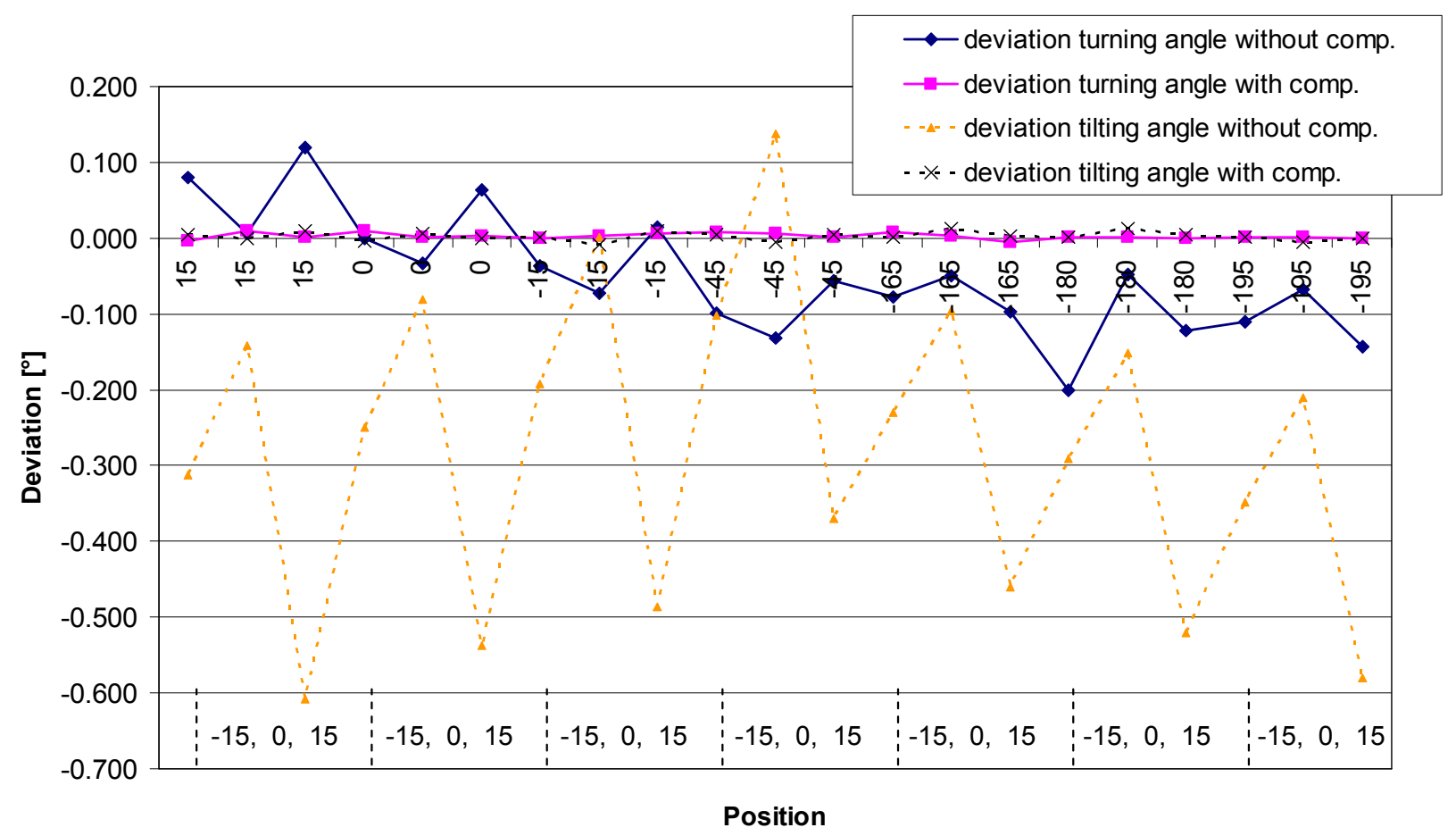

Figure 11: Deviations before and after Compensation

With the compensation procedure, a deviation $<0.012^{\circ}$ could be achieved in every position. This accuracy is sufficient for a precise machining. The duration of the procedure depends on the size of the error. The complete compensation takes between two and four seconds so that no appreciable non-productive time is added. The compensation time can be reduced further, if the robot speed is increased. Yet, a higher speed of the compensation movement can lead to larger deviations after compensation. If a lower target value is aspired (e.g. $0.04^{\circ}$ ) a compensation time smaller than two seconds can probably be reached. This will be investigated in further experiments.

For an automated compensation of the longitudinal deviations, the corresponding measuring system must be integrated into the clamping device first. This will be the aim of future work. Experiments in which the longitudinal error was measured manually have shown that a longitudinal accuracy of $\pm 0.025 \mathrm{~mm}$ is possible if the nominal value is approached gradually. Thus, the aspired accuracy should be achievable with the implemented measuring system. 


\section{Summary}

A new machine prototype enables the product flexible machining of three dimensionally curved extrusion profiles with small technical effort. In order to achieve a high machining quality, a precise positioning of the workpiece is necessary. It could be shown in experiments that the positioning accuracy shows a high repeatability. The absolute error must yet be reduced in order to reach the aspired accuracy. For this reason, a compensation approach was developed. After having send sensor data to the robot control, a corrected position is calculated and the error is compensated automatically. By this, the angular precision could be enhanced significantly. The following work will concentrate on implementing an automated longitudinal compensation. In a next step experiments on positioning and machining curved extrusions will be performed.

\section{Acknowledgement}

This paper is based on investigations of the collaborative research center SFB/TR10 which is kindly supported by the German Research Foundation (DFG).

\section{References}

[1] Fleischer, J.; Ruch, D.: Flexibles Spannen räumlich gekrümmter Profile - Form- und konturflexibles Spannen räumlich gekrümmter Aluminium-Strangpressprofile. in: wt-online, 09 (2005), pp. 712-716

[2] Fleischer, J.; Schmidt-Ewig, J.P.: Innovative Machine Kinematics for Combined Handling and Machining of Three-Dimensional Curved Lightweight Extrusion Structures. in: Annals of the CIRP, 54 (2005), pp. 317-320

[3] Fleischer, J.; Schmidt-Ewig, J. P.: Combination of a Parallel and a Serial Kinematics fort he Integrated Handling and Machining of Lightweight Extrusion Structures; In: 5th Chemnitz Parallel Kinematics Seminar, 05 (2006), pp. 289-306

[4] Fleischer, J.; Munzinger, C.; Schmidt-Ewig, J. P.: Kombinierte Handhabungs- und Bearbeitungskinematik. in: VDI Fortschritt-Berichte, 661 (2006), pp. 397-423

[5] Fleischer, J.; Stengel, G.: Bahngenerierung für eine robotergeführte Abtrenneinheit für räumlich gekrümmte Strangpressprofile. In: wt-online, 94 (2004), pp. 457-461

[6] Fleischer, J.; Munzinger, M.; Lamza, G.; Ruch, D. Schmidt-Ewig, J. P.; Stengel, G.; Schneider, M.: Maschinentechnik zur flexiblen Herstellung räumlich gekrümmter Strangpressprofile. In: ZWF - Zeitschrift für wirtschaftlichen Fabrikbetrieb, 7-8 (2006), pp. 426-430

[7] Shabana, A.A.: Computational Dynamics, John Wiley \& Sons, Inc., New York (2001) 